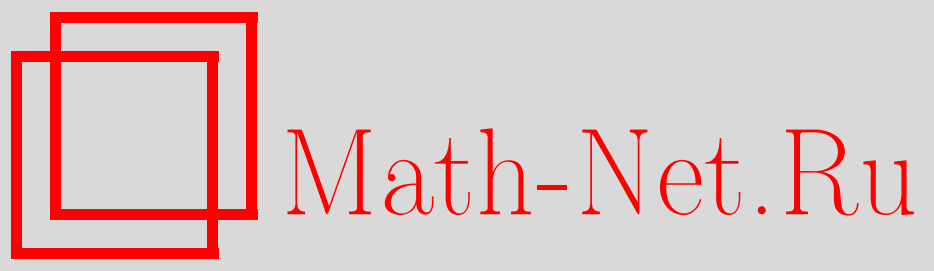

A.

A.

Логунов, М. А. Мествиришвили, Внешнее гравитационное поле нестатического сферически-симметричного тела в релятивистской теории гравитации, ТМФ, 2014, том 181, номер 2, 401-416

DOI: https://doi.org/10.4213/tmf8766

Использование Общероссийского математического портала Math-Net.Ru подразумевает, что вы прочитали и согласны с пользовательским соглашением http://www.mathnet.ru/rus/agreement

Параметры загрузки:

IP: 3.91 .87 .62

26 апреля 2023 г., 12:59:33

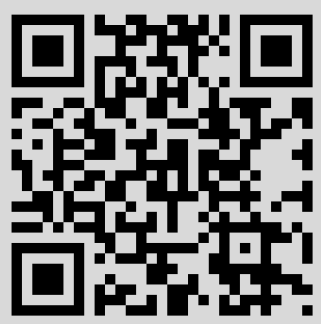




\section{ВНЕШНЕЕ ГРАВИТАЦИОННОЕ ПОЛЕ НЕСТАТИЧЕСКОГО СФЕРИЧЕСКИ-СИММЕТРИЧНОГО ТЕЛА В РЕЛЯТИВИСТСКОЙ ТЕОРИИ ГРАВИТАЦИИ}

\footnotetext{
Показано, что внешнее гравитационное поле нестатического сферически-симметричного тела является статическим.
}

Ключевые слова: метрический тензор Римана, гравитон, метрика пространства Минковского, инерциальная система.

\section{DOI: $10.4213 / \operatorname{tmf} 8766$}

В общей теории относительности (ОТО), которая связывает гравитационное поле с метрическим тензором риманова пространства, в классе допустимых функций доказана теорема Биркгофа, согласно которой внешнее поле нестатического сферически-симметричного тела может быть только статическим. В релятивистской теории гравитации (РТГ) гравитационное поле $\phi^{\mu \nu}$ является физическим полем в пространстве Минковского, источник которого - сохраняющийся в пространстве Минковского тензор энергии-импульса всех полей материи, включая и гравитационное поле. Такой подход приводит к эффективному риманову пространству и, соответственно, к системе уравнений, которая отличается от системы уравнений ОТО. Поэтому задача о нестатическом источнике требует специального рассмотрения. Этот вопрос нами изучался в статье [1], но, к сожалению, были допущены некоторые неточности, устранение которых и является целью данной работы. К тому же, в отличие от работы [1], настоящее рассмотрение имеет более общий характер.

Полная система уравнений РТГ имеет вид [2]

$$
\begin{gathered}
R^{\mu \nu}-\frac{1}{2} g^{\mu \nu} R+\frac{m^{2}}{2}\left[g^{\mu \nu}+\left(g^{\mu \alpha} g^{\nu \beta}-\frac{1}{2} g^{\mu \nu} g^{\alpha \beta}\right) \gamma_{\alpha \beta}\right]=8 \pi T^{\mu \nu}, \\
D_{\nu} \tilde{g}^{\nu \mu}=\partial_{\nu} \tilde{g}^{\nu \mu}+\gamma_{\alpha \beta}^{\mu} \tilde{g}^{\alpha \beta}=0 .
\end{gathered}
$$

Физическое гравитационное поле $\phi^{\mu \nu}$ определяется равенством

$$
\tilde{g}^{\mu \nu}=\tilde{\gamma}^{\mu \nu}+\tilde{\phi}^{\mu \nu}
$$

* Институт физики высоких энергий, Протвино, Московская обл., Россия. E-mail: Anatoly.Logunov@ihep.ru 
где $\tilde{g}^{\mu \nu}=\sqrt{-g} g^{\mu \nu}, \tilde{\gamma}^{\mu \nu}=\sqrt{-\gamma} \gamma^{\mu \nu}, \tilde{\phi}^{\mu \nu}=\sqrt{-\gamma} \phi^{\mu \nu}$. Оно обладает той же симметрией, что и метрический тензор риманова пространства $g^{\mu \nu}$.

В РТГ система координат определяется метрическим тензором $\gamma_{\mu \nu}$ пространства Минковского. В уравнении (1) присутствует масса покоя гравитона. Хорошо известно (см. [3], [4]), что введение массы покоя гравитона в линейные уравнения гравитационного поля приводит к следующей трудности: в этом случае, чтобы объяснить данные наблюдений по гравитационным эффектам в Солнечной системе, необходимо допустить, что сферически-симметричный нестатический источник испускает отрицательный поток энергии скалярных гравитонов, что физически недопустимо. Именно это обстоятельство и приводило к выводу, что масса покоя гравитона должна равняться нулю. Но так обстоит дело в линейной теории гравитации. В РТГ, как нелинейной теории, наличие массы покоя гравитона не порождает подобных трудностей.

Покажем, что в РТГ внешнее гравитационное поле нестатического сферически-симметричного тела точно так же, как и в ОТО, остается статическим, т.е. такое нестатическое тело не излучает гравитационные волны. Для нестатического сферически-симметричного тела интервал эффективного риманова пространства в общем случае имеет вид

$$
d s^{2}=B(\tau, r) d \tau^{2}-D(\tau, r) d r^{2}-2 E(\tau, r) d r d \tau-W^{2}(\tau, r)\left(d \theta^{2}+\sin ^{2} \theta d \phi^{2}\right) .
$$

Подберем некоторый интегрирующий множитель $\eta$ так, чтобы выражение

$$
d t=\eta(B d \tau-E d r)
$$

стало полным дифференциалом. Заметим, что число таких интегрирующих множителей бесконечно. Из выражения (4) находим

$$
d \tau=\frac{1}{\eta B} d t+\frac{E}{B} d r
$$

откуда следует, что существует функция $\alpha(t, r)$, дифференциал от которой равен

$$
d \tau=\dot{\alpha} d t+\dot{\alpha} d r
$$

где

$$
\dot{\alpha}=\frac{1}{\eta B}, \quad \dot{\alpha}=\frac{E}{B} .
$$

Заметим, что при преобразовании (5) мы не изменили систему отсчета, принятую в (3). Напомним, что все физически измеряемые величины должны быть хронометрически инвариантными величинами [5]. Подставляя (5) в интервал (3), получим

$$
d s^{2}=\frac{1}{\eta^{2} B} d t^{2}-d r^{2}\left(D+\frac{E^{2}}{B}\right)-W^{2}\left(d \theta^{2}+\sin ^{2} \theta d \phi^{2}\right) .
$$

После введения обозначений

$$
U=\frac{1}{\eta^{2} B}, \quad V=\left(D+\frac{E^{2}}{B}\right)
$$


выражение для $d s^{2}$ принимает вид

$$
d s^{2}=U(t, r) d t^{2}-V(t, r) d r^{2}-W^{2}(t, r)\left(d \theta^{2}+\sin ^{2} \theta d \phi^{2}\right)
$$

Функции $U, V$ и $W^{2}$ характеризуют гравитационное поле нестатического сферически-симметричного тела.

Таким образом, благодаря существованию интегрирующего множителя интервал в римановом пространстве для нестатического сферически-симметричного тела в общем случае имеет вид (9).

Для данной задачи, определяемой интервалом (9), интервал в инерциальной системе в пространстве Минковского в сферических координатах имеет вид

$$
d \sigma^{2}=d t^{2}-d r^{2}-r^{2}\left(d \theta^{2}+\sin ^{2} \theta d \phi^{2}\right)
$$

Ниже мы установим, что внешнее гравитационное поле вида (9), создаваемое нестатическим сферически-симметричным источником в инерциальной системе координат (10), может быть только статическим, т. е. метрические коэффициенты $U, V, W$ не зависят от времени $t$.

Из уравнений (1) для задачи, определяемой соотношениями (9) и (10), находим уравнения для функций $U, V$ и $W$ (см. приложение, формулы (П.28), (П.29) и $(П .31))$ :

$$
\begin{gathered}
\frac{1}{W^{2}}-\frac{1}{2 V} \frac{\partial}{\partial r}\left(\frac{1}{W^{2}} \frac{\partial W^{2}}{\partial r}\right)-\frac{3}{4 V W^{4}}\left(\frac{\partial W^{2}}{\partial r}\right)^{2}-\frac{\partial}{\partial r}\left(\frac{1}{2 V W^{2}} \frac{\partial W^{2}}{\partial r}\right)+ \\
+\frac{1}{2 U W^{2}} \frac{\partial W^{2}}{\partial t} \frac{\partial \ln (V W)}{\partial t}+\frac{m^{2}}{2}\left[1-\frac{r^{2}}{W^{2}}+\frac{1}{2}\left(\frac{1}{U}-\frac{1}{V}\right)\right]=0 \\
\frac{1}{W^{2}}+\frac{1}{2 U} \frac{\partial}{\partial t}\left(\frac{1}{W^{2}} \frac{\partial W^{2}}{\partial t}\right)+\frac{3}{4 U W^{4}}\left(\frac{\partial W^{2}}{\partial t}\right)^{2}+\frac{\partial}{\partial t}\left(\frac{1}{2 U W^{2}} \frac{\partial W^{2}}{\partial t}\right)- \\
-\frac{1}{2 V W^{2}} \frac{\partial W^{2}}{\partial r} \frac{\partial \ln (U W)}{\partial r}+\frac{m^{2}}{2}\left[1-\frac{r^{2}}{W^{2}}-\frac{1}{2}\left(\frac{1}{U}-\frac{1}{V}\right)\right]=0 \\
\frac{1}{W^{2}} \frac{\partial^{2} W^{2}}{\partial t \partial r}-\frac{1}{2 W^{4}} \frac{\partial W^{2}}{\partial r} \frac{\partial W^{2}}{\partial t}-\frac{1}{2 V W^{2}} \frac{\partial V}{\partial t} \frac{\partial W^{2}}{\partial r}-\frac{1}{2 U W^{2}} \frac{\partial U}{\partial r} \frac{\partial W^{2}}{\partial t}=0 .
\end{gathered}
$$

Уравнения (2) с учетом выражений (9) и (10) принимают вид (см. приложение, формулы (П.23), (П.24))

$$
W^{2}=\sqrt{\frac{U}{V}} q(r), \quad \frac{\partial}{\partial r}\left(W^{2} \sqrt{\frac{U}{V}}\right)=2 r \sqrt{U V},
$$

где $q(r)$ - произвольная положительная функция.

Так как согласно принципу причинности Гильберта $U>0, V>0, W^{2}>0$, для дальнейшего удобно перейти к новым обозначениям:

$$
U(t, r)=e^{\mu(t, r)}, \quad V(t, r)=e^{\nu(t, r)}, \quad W^{2}(t, r)=e^{\lambda(t, r)}, \quad q(r)=e^{\sigma(r)} .
$$


В переменных $\mu, \nu, \lambda, \sigma$ уравнения (11) имеют вид

$$
\begin{aligned}
e^{-\lambda}-e^{-\nu}\left(\lambda^{\prime \prime}+\frac{3}{4}\left(\lambda^{\prime}\right)^{2}-\frac{1}{2} \lambda^{\prime} \nu^{\prime}\right)+\frac{1}{2} e^{-\mu} \dot{\lambda}\left(\dot{\nu}+\frac{1}{2} \dot{\lambda}\right)+ \\
+\frac{m^{2}}{2}\left[1-r^{2} e^{-\lambda}+\frac{1}{2}\left(e^{-\mu}-e^{-\nu}\right)\right]=0 \\
e^{-\lambda}+e^{-\mu}\left(\ddot{\lambda}+\frac{3}{4}(\dot{\lambda})^{2}-\frac{1}{2} \dot{\lambda} \dot{\mu}\right)-\frac{1}{2} e^{-\nu} \lambda^{\prime}\left(\mu^{\prime}+\frac{1}{2} \lambda^{\prime}\right)+ \\
+\frac{m^{2}}{2}\left[1-r^{2} e^{-\lambda}-\frac{1}{2}\left(e^{-\mu}-e^{-\nu}\right)\right]=0 \\
\dot{\lambda}^{\prime}+\frac{1}{2} \dot{\lambda} \lambda^{\prime}-\frac{1}{2} \dot{\nu} \lambda^{\prime}-\frac{1}{2} \dot{\lambda} \mu^{\prime}=0
\end{aligned}
$$

где, например, $\dot{\lambda}=\partial \lambda / \partial t, \lambda^{\prime}=\partial \lambda / \partial r$. Уравнения (12) принимают вид

$$
\begin{gathered}
\lambda-\frac{1}{2}(\mu-\nu)=\sigma(r), \\
\mu^{\prime}-\nu^{\prime}+\sigma^{\prime}=2 r e^{\nu-\lambda} .
\end{gathered}
$$

Введем следующие обозначения:

$$
\begin{gathered}
2 \omega=\mu+\nu, \\
f=\lambda-\sigma(r) .
\end{gathered}
$$

Согласно (16) имеем

$$
\mu-\nu=2 f
$$

Из (18) и (20) находим

$$
\mu=\omega+f, \quad \nu=\omega-f .
$$

Запишем уравнение (17) через функции $\omega, f$ и $\sigma$ :

$$
2 f^{\prime}+\sigma^{\prime}=2 r e^{\omega-2 f-\sigma} .
$$

Дифференцируя (22) по $t$, находим

$$
2 \dot{f}^{\prime}=\left(2 f^{\prime}+\sigma^{\prime}\right)(\dot{\omega}-2 \dot{f})
$$

Подставляя это выражение в уравнение (15) и учитывая (19), (21), получим следующее неоднородное линейное уравнение в частных производных:

$$
\frac{\partial \omega}{\partial t} f^{\prime}-\frac{\partial \omega}{\partial r} \dot{f}=3 \dot{f} f^{\prime}
$$


Система обыкновенных дифференциальных уравнений, соответствующая уравнению (23), имеет вид

$$
\frac{d t}{f^{\prime}}=\frac{d r}{-\dot{f}}=\frac{d \omega}{3 \dot{f} f^{\prime}}
$$

Отсюда находим

$$
d \omega=3 \dot{f} d t, \quad d \omega=-3 f^{\prime} d r .
$$

Складывая эти равенства, получим

$$
d \omega=\frac{3}{2} \frac{\partial f}{\partial t} d t-\frac{3}{2} \frac{\partial f}{\partial r} d r .
$$

Из свойств полного дифференциала находим

$$
\frac{\partial^{2} f}{\partial t \partial r}=0
$$

но это равенство означает, что функция $f$ представима в виде

$$
f(t, r)=\psi(t)+\varphi(r)
$$

Общее решение уравнения (23) имеет вид

$$
\omega(t, r)=\frac{3}{2}(\psi(t)-\varphi(r))+F(f)
$$

где $F$ - произвольная функция.

С учетом выражений (24) и (25) уравнение (22) принимает следующий вид:

$$
2 \varphi^{\prime}+\sigma^{\prime}=2 r \exp \left[-\frac{1}{2} \psi(t)-\frac{7}{2} \varphi(r)+F(f)-\sigma\right] .
$$

Левая часть этого уравнения не зависит от $t$, поэтому правая часть также не должна зависеть от $t$. Это возможно, если $\psi(t)=$ const; тогда функции $\mu, \nu, \lambda$ не будут зависеть от времени. В этом случае гравитационное поле вида (9) является статическим. Но возможен и другой случай, когда $F=f / 2$. Тогда

$$
\omega(t, r)=2 \psi(t)-\varphi(r)
$$

а функции $\mu, \nu, \lambda$ согласно (21) и (19) будут иметь вид

$$
\mu=3 \psi(t), \quad \nu(t, r)=\psi(t)-2 \varphi(r), \quad \lambda(t, r)=\psi(t)+\varphi(r)+\sigma(r)=f+\sigma .
$$

Так мы пришли к интервалу, в котором в метрических коэффициентах переменные $t$ и $r$ разделены, а метрический коэффициент $U$ зависит только от времени. В этом случае уравнение (26) принимает вид

$$
2 \varphi^{\prime}+\sigma^{\prime}=2 r e^{-3 \varphi(r)-\sigma(r)} .
$$


Для анализа данного случая нам необходимо обратиться к уравнениям (13) и (14). Разность этих уравнений дает

$$
\begin{aligned}
& e^{-\nu}\left[-\lambda^{\prime \prime}-\frac{1}{2}\left(\lambda^{\prime}\right)^{2}+\frac{1}{2} \lambda^{\prime}\left(\mu^{\prime}+\nu^{\prime}\right)\right]+ \\
& \quad+e^{-\mu}\left[-\ddot{\lambda}-\frac{1}{2}(\dot{\lambda})^{2}+\frac{1}{2} \dot{\lambda}(\dot{\mu}+\dot{\nu})\right]+\frac{m^{2}}{2}\left(e^{-\mu}-e^{-\nu}\right)=0
\end{aligned}
$$

а их сумма -

$$
\begin{aligned}
& 2 e^{-\lambda}-e^{-\nu}\left[\lambda^{\prime \prime}+\left(\lambda^{\prime}\right)^{2}-\frac{1}{2} \lambda^{\prime}\left(\nu^{\prime}-\mu^{\prime}\right)\right]+ \\
& \quad+e^{-\mu}\left[\ddot{\lambda}+(\dot{\lambda})^{2}-\frac{1}{2} \dot{\lambda}(\dot{\mu}-\dot{\nu})\right]+m^{2}\left(1-r^{2} e^{-\lambda}\right)=0 .
\end{aligned}
$$

Согласно (16) имеем

$$
(\dot{\lambda})^{2}-\frac{1}{2} \dot{\lambda}(\dot{\mu}-\dot{\nu})=0
$$

поэтому уравнение (29) несколько упрощается:

$$
2 e^{-\lambda}-e^{-\nu}\left[\lambda^{\prime \prime}+\left(\lambda^{\prime}\right)^{2}-\frac{1}{2} \lambda^{\prime}\left(\nu^{\prime}-\mu^{\prime}\right)\right]+e^{-\mu} \ddot{\lambda}+m^{2}\left(1-r^{2} e^{-\lambda}\right)=0 .
$$

В уравнениях (28) и (30) переменные $t$ и $r$ разделяются:

$$
\begin{gathered}
-\varphi^{\prime \prime}-\sigma^{\prime \prime}-\frac{3}{2} \dot{\varphi}^{2}-\frac{1}{2} \hat{\sigma}^{2}-2 \dot{\varphi} \dot{\sigma}-\frac{m^{2}}{2}=k e^{-2 \varphi}, \\
\ddot{\psi}-\frac{3}{2} \dot{\psi}^{2}-\frac{m^{2}}{2}=k e^{2 \psi} \\
\varphi^{\prime \prime}+\sigma^{\prime \prime}+2 \dot{\varphi}^{2}+\dot{\sigma}^{2}+3 \dot{\varphi} \dot{\sigma}-2 e^{-3 \varphi-\sigma}\left(1-\frac{m^{2} r^{2}}{2}\right)=p e^{-2 \varphi}, \\
\ddot{\psi}+m^{2} e^{3 \psi}=p e^{2 \psi}
\end{gathered}
$$

Заметим, что системе уравнений (22), (31) и (33) удовлетворяет следующее решение:

$$
\varphi=0, \quad \sigma=\ln r^{2}, \quad k=-\frac{m^{2}}{2}, \quad p=m^{2}
$$

Обратимся к уравнениям (32) и (34). Введем новую переменную $\psi(t)=\ln a^{2}$. Уравнения (32) и (34) принимают вид

$$
\begin{aligned}
& 2 a \ddot{a}-8 \dot{a}^{2}-\frac{m^{2}}{2} a^{2}=k a^{6}, \\
& 2 a \ddot{a}-2 \dot{a}^{2}+m^{2} a^{8}=p a^{6} .
\end{aligned}
$$

Отсюда находим

$$
\dot{a}^{2}=-\frac{1}{12}\left[m^{2} a^{2}+2 m^{2} a^{8}+2(k-p) a^{6}\right] .
$$


Дифференцируя, получим

$$
\ddot{a}=-\frac{1}{24}\left[2 m^{2} a+16 m^{2} a^{7}+12(k-p) a^{5}\right] .
$$

Подставляя (38) и (39) в уравнение (36), находим соотношение между постоянными разделения переменных:

$$
p=-2 k \text {. }
$$

Введем новую переменную времени $d \tau=a^{3} d t$, тогда уравнение (38) принимает вид

$$
\frac{1}{a^{2}}\left(\frac{d a}{d \tau}\right)^{2}=\frac{m^{2}}{12}\left(-\frac{1}{a^{6}}-2+\frac{3}{\beta^{4} a^{2}}\right)
$$

где принято обозначение

$$
|k|=\frac{m^{2}}{2 \beta^{4}} .
$$

Уравнение (39) при этом принимает вид

$$
\frac{1}{a} \frac{d^{2} a}{d \tau^{2}}=-\frac{m^{2}}{6}\left(1-\frac{1}{a^{6}}\right) .
$$

Уравнение (41) имеет нетривиальное решение при условии $\beta<1$. Решение это будет иметь осциллирующий характер, и масштабный фактор а будет изменяться в пределах $a_{\min } \leqslant a \leqslant a_{\max }$. Поскольку в точке остановки $a_{\max }$ ускорение должно быть отрицательным, то согласно (43) имеет место неравенство $a_{\max }>1$.

С учетом выражений (27) интервал для нашего случая принимает вид

$$
d s^{2}=a^{2}\left[a^{4} d t^{2}-e^{-2 \varphi} d r^{2}-e^{\varphi+\sigma}\left(d \theta^{2}+\sin ^{2} \theta d \phi^{2}\right)\right] .
$$

Заметим, что для интервала (44) гармоническое уравнение (12) вне тела не зависит от временно́й переменной. Оно имеет вид

$$
\frac{d}{d r}\left(e^{2 \varphi+\sigma}\right)=2 r e^{-\varphi}
$$

В интервале (44) в квадратных скобках трехмерная пространственная часть определяется только функциями $\varphi(r)$ и $\sigma(r)$. Поскольку интервал (44) выражает внешнее гравитационное поле нестатического сферически-симметричного тела в отсутствие волнового процесса и потока энергии, физическое решение для функций $\varphi$ и $\sigma$ на пространственной бесконечности, где статическое поле также отсутствует, должно приводить к геометрии Евклида в сферических координатах, т. е. должны иметь место пределы

$$
\lim _{r \rightarrow \infty} \varphi(r)=0, \quad \lim _{r \rightarrow \infty} e^{\sigma(r)}=r^{2} .
$$


Эти два условия связаны между собой уравнением (45). Запишем уравнения (31) и (33) в более компактной форме, выразив их через компоненты тензора кривизны трехмерного пространства. Они принимают вид (см. приложение, формулы (П.39) и $(\Pi .40))$

$$
\begin{gathered}
R_{121}^{2}=\frac{1}{2} e^{-2 \varphi}\left(k+\frac{m^{2}}{2} e^{2 \varphi}\right), \\
R_{212}^{1}+R_{232}^{3}=e^{\varphi+\sigma}\left(k+\frac{m^{2} r^{2}}{2} e^{-(\varphi+\sigma)}\right) .
\end{gathered}
$$

Поскольку на пространственной бесконечности компоненты тензора кривизны равны нулю, из выражений (47) с учетом (46) находим параметр разделения переменных

$$
k=-\frac{m^{2}}{2}
$$

Тогда из (42) следует, что $\beta=1$. Но согласно этому равенству уравнение (41) имеет только тривиальное решение $a=1$. Следовательно, мы опять пришли к статическому решению.

Таким образом, мы приходим к общему выводу: для нестатического сферически-симметричного источника в инерциальной системе координат метрические коэффициенты интервала внешнего гравитационного поля могут быть только статическими. Поэтому такой источник не излучает гравитационные волны [2]-[4], [6]-[9].

\section{ПРИЛОЖЕНИЕ}

Интервал эффективного риманова пространства-времени для нестатического сферически-симметричного тела имеет общий вид (9). Отличные от нуля коэффициенты связности, соответствующие интервалу (9), даются выражениями

$$
\begin{gathered}
\Gamma_{00}^{0}=\frac{1}{2 U} \frac{\partial U}{\partial t}, \quad \Gamma_{01}^{0}=\frac{1}{2 U} \frac{\partial U}{\partial r}, \quad \Gamma_{11}^{0}=\frac{1}{2 U} \frac{\partial V}{\partial t}, \quad \Gamma_{22}^{0}=\frac{1}{2 U} \frac{\partial W^{2}}{\partial t} \\
\Gamma_{33}^{0}=\frac{1}{2 U} \frac{\partial W^{2}}{\partial t} \sin ^{2} \theta, \quad \Gamma_{00}^{1}=\frac{1}{2 V} \frac{\partial U}{\partial r}, \quad \Gamma_{01}^{1}=\frac{1}{2 V} \frac{\partial V}{\partial t}, \quad \Gamma_{11}^{1}=\frac{1}{2 V} \frac{\partial V}{\partial r}, \\
\Gamma_{22}^{1}=-\frac{1}{2 V} \frac{\partial W^{2}}{\partial r}, \quad \Gamma_{33}^{1}=-\frac{1}{2 V} \frac{\partial W^{2}}{\partial r} \sin ^{2} \theta, \quad \Gamma_{02}^{2}=\frac{1}{2 W^{2}} \frac{\partial W^{2}}{\partial t}, \\
\Gamma_{12}^{2}=\frac{1}{2 W^{2}} \frac{\partial W^{2}}{\partial r}, \quad \Gamma_{33}^{2}=-\sin \theta \cos \theta, \quad \Gamma_{03}^{3}=\frac{1}{2 W^{2}} \frac{\partial W^{2}}{\partial t}, \\
\Gamma_{13}^{3}=\frac{1}{2 W^{2}} \frac{\partial W^{2}}{\partial r}, \quad \Gamma_{23}^{3}=\operatorname{ctg} \theta .
\end{gathered}
$$

Интервал пространства Минковского в инерциальной системе в сферических координатах имеет вид (10), а отличные от нуля коэффициенты связности таковы:

$$
\gamma_{22}^{1}=-r, \quad \gamma_{33}^{1}=-r \sin ^{2} \theta, \quad \gamma_{12}^{2}=\gamma_{13}^{3}=\frac{1}{r}, \quad \gamma_{33}^{2}=-\sin \theta \cos \theta, \quad \gamma_{23}^{3}=\operatorname{ctg} \theta
$$


Используя для тензора Риччи представление

$$
R_{\sigma \nu}=\partial_{\lambda} \Gamma_{\sigma \nu}^{\lambda}-\partial_{\nu} \Gamma_{\sigma \lambda}^{\lambda}+\Gamma_{\sigma \nu}^{\tau} \Gamma_{\tau \lambda}^{\lambda}-\Gamma_{\sigma \lambda}^{\tau} \Gamma_{\nu \tau}^{\lambda}
$$

и учитывая (П.1), можно установить, что отличные от нуля компоненты $R_{\sigma \nu}$ имеют вид

$$
\begin{aligned}
& R_{00}=-\frac{1}{2 V} \frac{\partial^{2} V}{\partial t^{2}}+\frac{1}{2 V} \frac{\partial^{2} U}{\partial r^{2}}+\frac{1}{4 V^{2}}\left(\frac{\partial V}{\partial t}\right)^{2}-\frac{1}{4 U V}\left(\frac{\partial U}{\partial r}\right)^{2}- \\
& -\frac{1}{4 V^{2}} \frac{\partial U}{\partial r} \frac{\partial V}{\partial r}+\frac{1}{4 U V} \frac{\partial V}{\partial t} \frac{\partial U}{\partial t}-\frac{1}{W^{2}} \frac{\partial^{2} W^{2}}{\partial t^{2}}+\frac{1}{2 W^{4}}\left(\frac{\partial W^{2}}{\partial t}\right)^{2}+ \\
& +\frac{1}{2 U W^{2}} \frac{\partial U}{\partial t} \frac{\partial W^{2}}{\partial t}+\frac{1}{2 V W^{2}} \frac{\partial U}{\partial r} \frac{\partial W^{2}}{\partial r}, \\
& R_{11}=\frac{1}{2 U} \frac{\partial^{2} V}{\partial t^{2}}-\frac{1}{4 U^{2}} \frac{\partial U}{\partial t} \frac{\partial V}{\partial t}-\frac{1}{2 U} \frac{\partial^{2} U}{\partial r^{2}}+\frac{1}{4 U^{2}}\left(\frac{\partial U}{\partial r}\right)^{2}- \\
& -\frac{1}{W^{2}} \frac{\partial^{2} W^{2}}{\partial r^{2}}+\frac{1}{2 W^{4}}\left(\frac{\partial W^{2}}{\partial r}\right)^{2}+\frac{1}{2 U W^{2}} \frac{\partial V}{\partial t} \frac{\partial W^{2}}{\partial t}+\frac{1}{4 U V} \frac{\partial V}{\partial r} \frac{\partial U}{\partial r}+ \\
& +\frac{1}{2 V W^{2}} \frac{\partial V}{\partial r} \frac{\partial W^{2}}{\partial r}-\frac{1}{4 U V}\left(\frac{\partial V}{\partial t}\right)^{2}, \\
& R_{22}=-\frac{1}{2 V} \frac{\partial^{2} W^{2}}{\partial r^{2}}+\frac{1}{4 U V} \frac{\partial V}{\partial t} \frac{\partial W^{2}}{\partial t}+\frac{1}{4 V^{2}} \frac{\partial V}{\partial r} \frac{\partial W^{2}}{\partial r}+ \\
& +\frac{1}{2 U} \frac{\partial^{2} W^{2}}{\partial t^{2}}-\frac{1}{4 U^{2}} \frac{\partial U}{\partial t} \frac{\partial W^{2}}{\partial t}-\frac{1}{4 U V} \frac{\partial U}{\partial r} \frac{\partial W^{2}}{\partial r}+1 \\
& R_{33}=R_{22} \sin ^{2} \theta \\
& R_{01}=-\frac{1}{W^{2}} \frac{\partial^{2} W^{2}}{\partial r \partial t}+\frac{1}{2 W^{4}} \frac{\partial W^{2}}{\partial t} \frac{\partial W^{2}}{\partial r}+\frac{1}{2 U W^{2}} \frac{\partial U}{\partial r} \frac{\partial W^{2}}{\partial t}+ \\
& +\frac{1}{2 V W^{2}} \frac{\partial V}{\partial t} \frac{\partial W^{2}}{\partial r}
\end{aligned}
$$

а скалярная кривизна $R=g^{\sigma \nu} R_{\sigma \nu}$ имеет вид

$$
\begin{aligned}
R= & \frac{1}{U V} \frac{\partial^{2} U}{\partial r^{2}}-\frac{1}{2 V^{2} U} \frac{\partial V}{\partial r} \frac{\partial U}{\partial r}-\frac{1}{U V} \frac{\partial^{2} V}{\partial t^{2}}+\frac{1}{2 U V^{2}}\left(\frac{\partial V}{\partial t}\right)^{2}- \\
& -\frac{2}{U W^{2}} \frac{\partial^{2} W^{2}}{\partial t^{2}}+\frac{1}{2 U W^{4}}\left(\frac{\partial W^{2}}{\partial t}\right)^{2}+\frac{1}{2 V U^{2}} \frac{\partial U}{\partial t} \frac{\partial V}{\partial t}-\frac{1}{2 V U^{2}}\left(\frac{\partial U}{\partial r}\right)^{2}+ \\
& +\frac{2}{V W^{2}} \frac{\partial^{2} W^{2}}{\partial r^{2}}-\frac{1}{2 V W^{4}}\left(\frac{\partial W^{2}}{\partial r}\right)^{2}+\frac{1}{U^{2} W^{2}} \frac{\partial U}{\partial t} \frac{\partial W^{2}}{\partial t}- \\
& -\frac{1}{V^{2} W^{2}} \frac{\partial V}{\partial r} \frac{\partial W^{2}}{\partial r}+\frac{1}{U V W^{2}} \frac{\partial U}{\partial r} \frac{\partial W^{2}}{\partial r}-\frac{1}{U V W^{2}} \frac{\partial V}{\partial t} \frac{\partial W^{2}}{\partial t}-\frac{2}{W^{2}}
\end{aligned}
$$


Теперь найдем компоненты тензоров

$$
R_{\sigma \nu}-\frac{1}{2} g_{\sigma \nu} R, \quad R_{\nu}^{\mu}-\frac{1}{2} \delta_{\nu}^{\mu} R
$$

После подстановки (П.4) и (П.9) в тождество

$$
R_{00}-\frac{1}{2} g_{00} R \equiv R_{00}-\frac{1}{2} U R
$$

имеем

$$
\begin{aligned}
R_{00}-\frac{1}{2} U R= & \frac{U}{W^{2}}+\frac{U}{4 V W^{4}}\left(\frac{\partial W^{2}}{\partial r}\right)^{2}+\frac{U}{2 W^{2} V^{2}} \frac{\partial V}{\partial r} \frac{\partial W^{2}}{\partial r}+ \\
& +\frac{1}{2 V W^{2}} \frac{\partial V}{\partial t} \frac{\partial W^{2}}{\partial t}+\frac{1}{4 W^{4}}\left(\frac{\partial W^{2}}{\partial t}\right)^{2}-\frac{U}{V W^{2}} \frac{\partial^{2} W^{2}}{\partial r^{2}}
\end{aligned}
$$

Так как

$$
R_{0}^{0}-\frac{1}{2} R=\frac{1}{U}\left(R_{00}-\frac{1}{2} U R\right)
$$

для левой части (П.12) получим выражение

$$
\begin{aligned}
R_{0}^{0}-\frac{1}{2} R= & \frac{1}{W^{2}}+\frac{1}{4 V W^{4}}\left(\frac{\partial W^{2}}{\partial r}\right)^{2}+\frac{1}{2 V^{2} W^{2}} \frac{\partial V}{\partial r} \frac{\partial W^{2}}{\partial r}+ \\
& +\frac{1}{2 U V W^{2}} \frac{\partial V}{\partial t} \frac{\partial W^{2}}{\partial t}+\frac{1}{4 U W^{4}}\left(\frac{\partial W^{2}}{\partial t}\right)^{2}-\frac{1}{W^{2} V} \frac{\partial^{2} W^{2}}{\partial r^{2}}
\end{aligned}
$$

Аналогично, подставляя (П.5) и (П.9) в тождество

$$
R_{11}-\frac{1}{2} g_{11} R \equiv R_{11}+\frac{1}{2} V R
$$

найдем

$$
\begin{aligned}
R_{11}+\frac{1}{2} V R= & -V\left[\frac{1}{W^{2}}+\frac{1}{U W^{2}} \frac{\partial^{2} W^{2}}{\partial t^{2}}-\frac{1}{2 U W^{2} V} \frac{\partial W^{2}}{\partial r} \frac{\partial U}{\partial r}+\right. \\
& \left.+\frac{1}{2 U^{2} W^{2}} \frac{\partial W^{2}}{\partial t} \frac{\partial U}{\partial t}-\frac{1}{4 U W^{4}}\left(\frac{\partial W^{2}}{\partial t}\right)^{2}-\frac{1}{4 V W^{4}}\left(\frac{\partial W^{2}}{\partial r}\right)^{2}\right]
\end{aligned}
$$

Ho

$$
R_{1}^{1}-\frac{1}{2} R=-\frac{1}{V}\left(R_{11}+\frac{1}{2} V R\right)
$$


поэтому имеем

$$
\begin{aligned}
R_{1}^{1}-\frac{1}{2} R= & \frac{1}{W^{2}}+\frac{1}{U W^{2}} \frac{\partial^{2} W^{2}}{\partial t^{2}}-\frac{1}{2 U V W^{2}} \frac{\partial W^{2}}{\partial r} \frac{\partial U}{\partial r}- \\
& -\frac{1}{2 U^{2} W^{2}} \frac{\partial W^{2}}{\partial t} \frac{\partial U}{\partial t}-\frac{1}{4 U W^{4}}\left(\frac{\partial W^{2}}{\partial t}\right)^{2}-\frac{1}{4 V W^{4}}\left(\frac{\partial W^{2}}{\partial r}\right)^{2} .
\end{aligned}
$$

Нетрудно также установить, что для

$$
R_{22}+\frac{1}{2} W^{2} R
$$

после подстановки (П.6) и (П.9) получается выражение

$$
\begin{aligned}
R_{22} & +\frac{1}{2} W^{2} R=-W^{2}\left[\frac{1}{2 U W^{2}} \frac{\partial^{2} W^{2}}{\partial t^{2}}+\frac{1}{2 U V}\left(\frac{\partial^{2} V}{\partial t^{2}}-\frac{\partial^{2} U}{\partial r^{2}}\right)-\frac{1}{2 V W^{2}} \frac{\partial^{2} W^{2}}{\partial r^{2}}\right]- \\
& -\frac{W^{2}}{4}\left[\frac{1}{V W^{4}}\left(\frac{\partial W^{2}}{\partial r}\right)^{2}+\frac{1}{V^{2} W^{2}} \frac{\partial W^{2}}{\partial r} \frac{\partial V}{\partial r}-\frac{1}{V U^{2}}\left(\frac{\partial V}{\partial t} \frac{\partial U}{\partial t}-\left(\frac{\partial U}{\partial r}\right)^{2}\right)+\right. \\
& +\frac{1}{U V^{2}}\left(\frac{\partial U}{\partial r} \frac{\partial V}{\partial r}-\left(\frac{\partial V}{\partial t}\right)^{2}\right)-\frac{1}{U^{2} W^{2}} \frac{\partial W^{2}}{\partial t} \frac{\partial U}{\partial t}-\frac{1}{U W^{4}}\left(\frac{\partial W^{2}}{\partial t}\right)^{2}- \\
& \left.-\frac{1}{U V W^{2}}\left(\frac{\partial W^{2}}{\partial r} \frac{\partial U}{\partial r}-\frac{\partial W^{2}}{\partial t} \frac{\partial V}{\partial t}\right)\right],
\end{aligned}
$$

а для

$$
R_{2}^{2}-\frac{1}{2} R=-\frac{1}{W^{2}}\left(R_{22}+\frac{1}{2} W^{2} R\right)
$$

- выражение

$$
\begin{aligned}
R_{2}^{2} & -\frac{1}{2} R=\frac{1}{2}\left[\frac{1}{U W^{2}} \frac{\partial^{2} W^{2}}{\partial t^{2}}+\frac{1}{U V}\left(\frac{\partial^{2} V}{\partial t^{2}}-\frac{\partial^{2} U}{\partial r^{2}}\right)-\frac{1}{V W^{2}} \frac{\partial^{2} W^{2}}{\partial r^{2}}\right]+ \\
+ & \frac{1}{4}\left[\frac{1}{V W^{4}}\left(\frac{\partial W^{2}}{\partial r}\right)^{2}+\frac{1}{V^{2} W^{2}} \frac{\partial W^{2}}{\partial r} \frac{\partial V}{\partial r}-\frac{1}{V U^{2}}\left(\frac{\partial V}{\partial t} \frac{\partial U}{\partial t}-\left(\frac{\partial U}{\partial r}\right)^{2}\right)+\right. \\
+ & \frac{1}{U V^{2}}\left(\frac{\partial U}{\partial r} \frac{\partial V}{\partial r}-\left(\frac{\partial V}{\partial t}\right)^{2}\right)-\frac{1}{U^{2} W^{2}} \frac{\partial W^{2}}{\partial t} \frac{\partial U}{\partial t}- \\
- & \left.\frac{1}{U W^{4}}\left(\frac{\partial W^{2}}{\partial t}\right)^{2}-\frac{1}{U V W^{2}}\left(\frac{\partial W^{2}}{\partial r} \frac{\partial U}{\partial r}-\frac{\partial W^{2}}{\partial t} \frac{\partial V}{\partial t}\right)\right] .
\end{aligned}
$$

Наконец, согласно (П.8) для $R_{1}^{0}$ имеем

$$
\begin{aligned}
R_{1}^{0}=\frac{1}{U} R_{01}= & -\frac{1}{U}\left(\frac{1}{W^{2}} \frac{\partial^{2} W^{2}}{\partial t \partial r}-\frac{1}{2 W^{4}} \frac{\partial W^{2}}{\partial t} \frac{\partial W^{2}}{\partial r}-\right. \\
& \left.-\frac{1}{2 U W^{2}} \frac{\partial U}{\partial r} \frac{\partial W^{2}}{\partial t}-\frac{1}{2 V W^{2}} \frac{\partial V}{\partial t} \frac{\partial W^{2}}{\partial r}\right)
\end{aligned}
$$


Введем обозначение

$$
M_{\nu}^{\mu}=\frac{m^{2}}{2}\left(\delta_{\nu}^{\mu}+g^{\mu \alpha} \gamma_{\alpha \nu}-\frac{1}{2} \delta_{\nu}^{\mu} g^{\alpha \beta} \gamma_{\alpha \beta}\right)
$$

Так как

$$
g^{00}=\frac{1}{U}, \quad g^{11}=-\frac{1}{V}, \quad g^{22}=-\frac{1}{W^{2}}, \quad g^{33}=-\frac{1}{W^{2} \sin ^{2} \theta}
$$

отличные от нуля компоненты $M_{\nu}^{\mu}$ имеют вид

$$
\begin{aligned}
& M_{0}^{0}=\frac{m^{2}}{2}\left[1-\frac{r^{2}}{W^{2}}+\frac{1}{2}\left(\frac{1}{U}-\frac{1}{V}\right)\right], \\
& M_{1}^{1}=\frac{m^{2}}{2}\left[1-\frac{r^{2}}{W^{2}}-\frac{1}{2}\left(\frac{1}{U}-\frac{1}{V}\right)\right], \\
& M_{2}^{2}=M_{3}^{3}=\frac{m^{2}}{2}\left[1-\frac{1}{2}\left(\frac{1}{U}+\frac{1}{V}\right)\right] .
\end{aligned}
$$

Принимая во внимание (П.20) и равенство

$$
\sqrt{-g}=\sqrt{U V} W^{2} \sin \theta
$$

для компонент тензорной плотности $\tilde{g}^{\mu \nu}=\sqrt{-g} g^{\mu \nu}$ находим

$$
\begin{gathered}
\tilde{g}^{00}=\sqrt{\frac{V}{U}} W^{2} \sin \theta, \quad \tilde{g}^{11}=-\sqrt{\frac{U}{V}} W^{2} \sin \theta, \quad \tilde{g}^{22}=-\sqrt{U V} \sin \theta, \\
\tilde{g}^{33}=-\sqrt{U V} \frac{1}{\sin \theta}, \quad \tilde{g}^{\mu \nu}=0 \quad \text { при } \quad \mu \neq \nu .
\end{gathered}
$$

Используя (П.2) и (П.22) в уравнении (2), получим

$$
\frac{\partial}{\partial t}\left(\sqrt{\frac{V}{U}} W^{2}\right)=0
$$

и

$$
\frac{\partial}{\partial r}\left(\sqrt{\frac{U}{V}} W^{2}\right)=2 r \sqrt{U V}
$$

Из (П.23) очевидно, что

$$
\sqrt{\frac{V}{U}} W^{2}=q(r)
$$

где $q(r)$ - положительная зависящая только от $r$ произвольная функция. 
Запишем (П.13) и (П.15) в несколько ином виде. Приведем четыре члена из (П.13) к следующему виду:

$$
\begin{aligned}
\frac{1}{W^{2}} & -\frac{1}{W^{2} V} \frac{\partial^{2} W^{2}}{\partial r^{2}}+\frac{1}{4 V W^{4}}\left(\frac{\partial W^{2}}{\partial r}\right)^{2}+\frac{1}{2 W^{2} V^{2}} \frac{\partial V}{\partial r} \frac{\partial W^{2}}{\partial r}= \\
& =\frac{1}{W^{2}}-\frac{1}{2 V} \frac{\partial}{\partial r}\left(\frac{1}{W^{2}} \frac{\partial W^{2}}{\partial r}\right)-\frac{3}{4} \frac{1}{V W^{4}}\left(\frac{\partial W^{2}}{\partial r}\right)^{2}-\frac{\partial}{\partial r}\left(\frac{1}{2 V W^{2}} \frac{\partial W^{2}}{\partial r}\right)
\end{aligned}
$$

а оставшиеся два члена - к виду

$$
\frac{1}{2 U V W^{2}} \frac{\partial V}{\partial t} \frac{\partial W^{2}}{\partial t}+\frac{1}{4 U W^{4}}\left(\frac{\partial W^{2}}{\partial t}\right)^{2}=\frac{1}{2 U W^{2}} \frac{\partial W^{2}}{\partial t} \frac{\partial}{\partial t} \ln (V W)
$$

Тогда

$$
\begin{aligned}
R_{0}^{0}-\frac{1}{2} R= & \frac{1}{W^{2}}-\frac{1}{2 V} \frac{\partial}{\partial r}\left(\frac{1}{W^{2}} \frac{\partial W^{2}}{\partial r}\right)-\frac{3}{4} \frac{1}{V W^{4}}\left(\frac{\partial W^{2}}{\partial r}\right)^{2}- \\
& -\frac{\partial}{\partial r}\left(\frac{1}{2 V W^{2}} \frac{\partial W^{2}}{\partial r}\right)+\frac{1}{2 U W^{2}} \frac{\partial W^{2}}{\partial t} \frac{\partial}{\partial t} \ln (V W)
\end{aligned}
$$

Аналогично четыре члена из (П.15) представим в следующем виде:

$$
\begin{aligned}
\frac{1}{W^{2}} & +\frac{1}{U W^{2}} \frac{\partial^{2} W^{2}}{\partial t^{2}}-\frac{1}{4 U W^{4}}\left(\frac{\partial W^{2}}{\partial t}\right)^{2}-\frac{1}{2 W^{2} U^{2}} \frac{\partial W^{2}}{\partial t} \frac{\partial U}{\partial t}= \\
& =\frac{1}{W^{2}}+\frac{1}{2 U} \frac{\partial}{\partial t}\left(\frac{1}{W^{2}} \frac{\partial W^{2}}{\partial t}\right)+\frac{3}{4 U W^{4}}\left(\frac{\partial W^{2}}{\partial t}\right)^{2}+\frac{\partial}{\partial t}\left(\frac{1}{2 W^{2} U} \frac{\partial W^{2}}{\partial t}\right)
\end{aligned}
$$

а оставшиеся два члена - в виде

$$
-\frac{1}{2 U V W^{2}} \frac{\partial W^{2}}{\partial r} \frac{\partial U}{\partial r}-\frac{1}{4 V W^{4}}\left(\frac{\partial W^{2}}{\partial r}\right)^{2}=-\frac{1}{2 V W^{2}} \frac{\partial W^{2}}{\partial r} \frac{\partial}{\partial r} \ln (U W)
$$

Следовательно,

$$
\begin{aligned}
R_{1}^{1}-\frac{1}{2} R= & \frac{1}{W^{2}}+\frac{1}{2 U} \frac{\partial}{\partial t}\left(\frac{1}{W^{2}} \frac{\partial W^{2}}{\partial t}\right)+\frac{3}{4 U W^{4}}\left(\frac{\partial W^{2}}{\partial t}\right)^{2}+ \\
& +\frac{\partial}{\partial t}\left(\frac{1}{2 W^{2} U} \frac{\partial W^{2}}{\partial t}\right)-\frac{1}{2 V W^{2}} \frac{\partial W^{2}}{\partial r} \frac{\partial}{\partial r} \ln (U W)
\end{aligned}
$$


Учитывая выражения (П.17), (П.21), (П.26) и (П.27) в системе уравнений

$$
R_{\nu}^{\mu}-\frac{1}{2} \delta_{\nu}^{\mu} R+M_{\nu}^{\mu}=\varkappa T_{\nu}^{\mu},
$$

получим

$$
\begin{aligned}
R_{0}^{0} & -\frac{1}{2} R+M_{0}^{0}=\frac{1}{W^{2}}-\frac{1}{2 V} \frac{\partial}{\partial r}\left(\frac{1}{W^{2}} \frac{\partial W^{2}}{\partial r}\right)-\frac{3}{4 V W^{4}}\left(\frac{\partial W^{2}}{\partial r}\right)^{2}- \\
& -\frac{\partial}{\partial r}\left(\frac{1}{2 V W^{2}} \frac{\partial W^{2}}{\partial r}\right)+\frac{1}{2 U W^{2}} \frac{\partial W^{2}}{\partial t} \frac{\partial}{\partial t} \ln (V W)+ \\
& +\frac{m^{2}}{2}\left[1-\frac{r^{2}}{W^{2}}+\frac{1}{2}\left(\frac{1}{U}-\frac{1}{V}\right)\right]=\varkappa T_{0}^{0}, \\
R_{1}^{1} & -\frac{1}{2} R+M_{1}^{1}=\frac{1}{W^{2}}+\frac{1}{2 U} \frac{\partial}{\partial t}\left(\frac{1}{W^{2}} \frac{\partial W^{2}}{\partial t}\right)+\frac{3}{4 U W^{4}}\left(\frac{\partial W^{2}}{\partial t}\right)^{2}+ \\
& +\frac{\partial}{\partial t}\left(\frac{1}{2 U W^{2}} \frac{\partial W^{2}}{\partial t}\right)-\frac{1}{2 V W^{2}} \frac{\partial W^{2}}{\partial r} \frac{\partial}{\partial r} \ln (U W)+ \\
& +\frac{m^{2}}{2}\left[1-\frac{r^{2}}{W^{2}}-\frac{1}{2}\left(\frac{1}{U}-\frac{1}{V}\right)\right]=\varkappa T_{1}^{1}, \\
R_{2}^{2}- & \frac{1}{2} R+M_{2}^{2}=\frac{1}{2}\left[\frac{1}{U W^{2}} \frac{\partial^{2} W^{2}}{\partial t^{2}}+\frac{1}{U V}\left(\frac{\partial^{2} V}{\partial t^{2}}-\frac{\partial^{2} U}{\partial r^{2}}\right)-\frac{1}{V W^{2}} \frac{\partial^{2} W^{2}}{\partial r^{2}}\right]+ \\
+ & \frac{1}{4}\left[\frac{1}{V W^{4}}\left(\frac{\partial W^{2}}{\partial r}\right)^{2}+\frac{1}{V^{2} W^{2}} \frac{\partial W^{2}}{\partial r} \frac{\partial V}{\partial r}-\frac{1}{V U^{2}}\left(\frac{\partial V}{\partial t} \frac{\partial U}{\partial t}-\left(\frac{\partial U}{\partial r}\right)^{2}\right)+\right. \\
+ & \frac{1}{U V^{2}}\left(\frac{\partial U}{\partial r} \frac{\partial V}{\partial r}-\left(\frac{\partial V}{\partial t}\right)^{2}\right)-\frac{1}{U V W^{2}}\left(\frac{\partial W^{2}}{\partial r} \frac{\partial U}{\partial r}-\frac{\partial V}{\partial t} \frac{\partial W^{2}}{\partial t}\right)- \\
- & \left.\frac{1}{U W^{4}}\left(\frac{\partial W^{2}}{\partial t}\right)^{2}-\frac{1}{U^{2} W^{2}} \frac{\partial W^{2}}{\partial t} \frac{\partial U}{\partial t}\right]+\frac{m^{2}}{2}\left[1-\frac{1}{2}\left(\frac{1}{U}+\frac{1}{V}\right)\right]=\varkappa T_{2}^{2} \\
-R_{1}^{0} & =\frac{1}{U W^{2}} \frac{\partial^{2} W^{2}}{\partial t \partial r}-\frac{1}{2 W^{4} U} \frac{\partial W^{2}}{\partial r} \frac{\partial W^{2}}{\partial t}-\frac{1}{2 U V W^{2}} \frac{\partial V}{\partial t} \frac{\partial W^{2}}{\partial r}- \\
& -\frac{1}{2 U^{2} W^{2}} \frac{\partial U}{\partial r} \frac{\partial W^{2}}{\partial t}=-\varkappa T_{1}^{0} . \\
&
\end{aligned}
$$

Согласно (44) квадрат элемента длины пространственной части интервала $d s^{2}$ имеет вид

$$
d l^{2}=e^{-2 \varphi} d r^{2}+e^{\varphi+\sigma}\left(d \theta^{2}+\sin ^{2} \theta d \phi^{2}\right) .
$$

Обозначим соответствующие метрические коэффициенты через $\eta_{m n}$, т. е.

$$
\eta_{11}=e^{-2 \varphi(r)}, \quad \eta_{22}=e^{\varphi(r)+\sigma(r)}, \quad \eta_{33}=\eta_{22} \sin ^{2} \theta, \quad \eta_{m n}=0 \quad \text { при } \quad m \neq n .
$$


Исходя из этих выражений нетрудно установить, что отличные от нуля компоненты связности

$$
\eta_{m n}^{l}=\frac{1}{2} \eta^{l k}\left(\partial_{m} \eta_{k n}+\partial_{n} \eta_{k m}-\partial_{k} \eta_{m n}\right)
$$

имеют вид

$$
\begin{aligned}
& \eta_{11}^{1}=-\varphi^{\prime}(r), \quad \eta_{22}^{1}=-\frac{1}{2}\left(\varphi^{\prime}+\sigma^{\prime}\right) e^{3 \varphi+\sigma}, \quad \eta_{33}^{1}=\eta_{22}^{1} \sin ^{2} \theta \\
& \eta_{12}^{2}=\eta_{13}^{3}=\frac{1}{2}\left(\varphi^{\prime}+\sigma^{\prime}\right), \quad \eta_{33}^{2}=-\sin \theta \cos \theta, \quad \eta_{23}^{3}=\operatorname{ctg} \theta
\end{aligned}
$$

Очевидно, что

$$
\eta_{1 p}^{p}=\sigma^{\prime}, \quad \eta_{2 p}^{p}=\operatorname{ctg} \theta, \quad \eta_{3 p}^{p}=0
$$

Для компонент тензора кривизны трехмерного пространства

$$
R_{m n p}^{k}=\partial_{n} \eta_{m p}^{k}-\partial_{p} \eta_{m n}^{k}+\eta_{m p}^{e} \eta_{e n}^{k}-\eta_{m n}^{e} \eta_{e p}^{k}
$$

получаем выражения

$$
\begin{gathered}
R_{212}^{1}=-\frac{1}{2} e^{3 \varphi+\sigma}\left[\varphi^{\prime \prime}+\sigma^{\prime \prime}+\frac{1}{2}\left(\varphi^{\prime}+\sigma^{\prime}\right)\left(3 \varphi^{\prime}+\sigma^{\prime}\right)\right] \\
R_{313}^{1}=R_{212}^{1} \sin ^{2} \theta \\
R_{121}^{2}=-\frac{1}{2}\left[\varphi^{\prime \prime}+\sigma^{\prime \prime}+\frac{1}{2}\left(\varphi^{\prime}+\sigma^{\prime}\right)\left(3 \varphi^{\prime}+\sigma^{\prime}\right)\right], \\
R_{323}^{2}=\left[1-\frac{1}{4}\left(\varphi^{\prime}+\sigma^{\prime}\right)^{2} e^{3 \varphi+\sigma}\right] \sin ^{2} \theta, \quad R_{131}^{3}=R_{121}^{2}, \\
R_{232}^{3}=1-\frac{1}{4}\left(\varphi^{\prime}+\sigma^{\prime}\right)^{2} e^{3 \varphi+\sigma} .
\end{gathered}
$$

Учитывая (П.37) в уравнении (31), получим

$$
R_{121}^{2}=\frac{1}{2} e^{-2 \varphi}\left(k+\frac{m^{2}}{2} e^{2 \varphi}\right)
$$

Аналогично, складывая (П.35) и (П.38) и принимая во внимание (40), уравнение (33) можно представить в виде

$$
R_{212}^{1}+R_{232}^{3}=e^{\varphi+\sigma}\left(k+\frac{m^{2} r^{2}}{2} e^{-(\varphi+\sigma)}\right) .
$$

Благодарности. Авторы выражают благодарность С. С. Герштейну, В. А. Петрову, А. П. Самохину, Н. Е. Тюрину за внимание и ценные обсуждения. 


\section{Список литературы}

[1] А. А. Логунов, М. А. Мествиришвили, ТМФ, 145:3 (2005), 425-432.

[2] А. А. Логунов, Релятивистская теория гравитации, М., Наука, 2012.

[3] В. И. Захаров, Писъма в ЖЭТФ, 12:9 (1970), 447-449.

[4] H. van Dam, M. Veltman, Nucl. Phys. B, 22:2 (1970), 397-411.

[5] А. Л. Зельманов, В. Г. Агаков, Элементы общей теории относительности, М., Наука, 1989.

[6] Ю. М. Лоскутов, Вестн. Моск. ун-та. Сер. 3. Физика. Астрономия, 32:4 (1991), 49-54.

[7] Ю. М. Лоскутов, ТМФ, 107:2 (1996), 329-343.

[8] Yu. M. Loskutov, Proceedings of the Sixth Marcel Grossmann Meeting on General Relativity. Part B (Kyoto, Japan, June 23-29, 1991), eds. H. Sato, T. Nakamura, World Sci., Singapore, 1992, 1658-1660.

[9] С. С. Герштейн, А. А. Логунов, М. А. Мествиришвили, ТМФ, 160:2 (2009), 270-275.

Поступила в редакцию 8.07.2014 